Revue internationale P.M.E.

Économie et gestion de la petite et moyenne entreprise

\title{
Les conditions de la création et du développement des PME en Pologne
}

\section{Aleksandra Jewtuchowicz}

Volume 5, numéro 1, 1992

URI : https://id.erudit.org/iderudit/1008132ar

DOI : https://doi.org/10.7202/1008132ar

Aller au sommaire du numéro

\section{Éditeur(s)}

Presses de l’Université du Québec

ISSN

0776-5436 (imprimé)

1918-9699 (numérique)

Découvrir la revue

Citer cet article

Jewtuchowicz, A. (1992). Les conditions de la création et du développement des PME en Pologne. Revue internationale P.M.E., 5(1), 43-62.

https://doi.org/10.7202/1008132ar

\section{Résumé de l'article}

Cet article décrit les opportunités et les obstacles au développement des petites et des moyennes entreprises dans le contexte de la restructuration de l'économie polonaise et des exigences imposées par la nouvelle économie mondiale. L'examen de la structure industrielle et des conditions économiques qui prévalent à Lodz, seconde ville de Pologne, sert d'exemple pour illustrer la situation.

L'expansion des petites entreprises, actuellement marginales, constitue l'une des voies possibles à cette restructuration de l'économie. En plus des difficultés conjoncturelles et systémiques, des obstacles liés au manque de capitaux, à la difficulté de trouver un local et au manque d'esprit d'entreprise et de formation constituent des entraves à cette expansion.

Un engagement plus grand et une assistance plus concrète des autorités régionales et des organes du gouvernement, surtout en ce qui concerne la reconversion professionnelle et la promotion d'un environnement entrepreneurial propice, devraient favoriser le développement et l'expansion des PME en Pologne. 


\title{
Les conditions de la création et du développement des PME en Pologne
}

\author{
Aleksandra JEWTUCHOWICZ \\ Université de Lodz \\ Institut de la politique régionale \\ Pologne
}

\begin{abstract}
RÉSUMÉ
Cet article décrit les opportunités et les obstacles au développement des petites et des moyennes entreprises dans le contexte de la restructuration de l'économie polonaise et des exigences imposées par la nouvelle économie mondiale. L'examen de la structure industrielle et des conditions économiques qui prévalent à Lodz, seconde ville de Pologne, sert d'exemple pour illustrer la situation.

L'expansion des petites entreprises, actuellement marginales, constitue l'une des voies possibles à cette restructuration de l'économie. En plus des difficultés conjoncturelles et systémiques, des obstacles liés au manque de capitaux, à la difficulté de trouver un local et au manque d'esprit d'entreprise et de formation constituent des entraves à cette expansion.

Un engagement plus grand et une assistance plus concrète des autorités régionales et des organes du gouvernement, surtout en ce qui concerne la reconversion professionnelle et la promotion d'un environnement entrepreneurial propice, devraient favoriser le développement et l'expansion des PME en Pologne.
\end{abstract}

- Aleksandra Jewtuchowicz, docteure ès sciences économique, est professeure, responsable du Département d'économie régionale et environnement à l'Université de Lodz. Elle est spécialiste en planification et économie régionale et urbaine, économie industrielle et gestion. Ses thèmes de recherche sont : les mutations socioéconomiques et le développement local ; les conditions de création et de développement des PME-PMI ; les politiques locales de développement économique ; le développement régional. Publications récentes : Les Statistiques Régionales et Urbaines, 1989.

Adresse : Université de Lodz, Département d'économie régionale et environnement, 90-248 Lodz, Pologne, ul. Polskiej Org. Wojskowej 19/5. 


\begin{abstract}
This article define the opportunities and obstacles encountered by small and medium-sized businesses within the context of the restructurization of the Polish economy and the demands placed on it by a new world ecomonic order.

The growth of small business, now marginal, is one possible way toward this restructurization. Added to conjunctural and systemical difficulties which hinder this expansion, are the osbtacles created by a lack of capital, difficulty in finding office space and the absence of an entrepreneurship culture, and training.

A stronger commitment and more concrete assistance on the part of regional authorities and government agencies especially concerning professional reconversion and the promotion of a favourable entrepreneurial environment, would encourage the development and growth of SMBs in Poland.
\end{abstract}

\title{
RESUMEN
}

Este artículo presenta las oportutinades y los obstáculos al desarrollo de pequeñas y medianas empresas (PME) dentro el contexto de la reestructuración de la economía polonesa y de las exigencias impuestas por la nueva economia mundial. El exámen de la estructura industrial y de las condiciónes económicas que rigen a Lodz, la segunda ciudad de Polonia, sirve de ejemplo para ilustrar la situación.

La expansión de las pequeñas empresas, actualmente mariginales, constituye una de las vías posibles a esta reestructuración. Ademas de las dificultades conyuturales y sistemáticas, los obstáculos ligados a la falta de capital, a la dificultad de encontrar un localy a la falta de espiritu de empresa y la formacion corepondiente constituyen las travas a ésta expansión.

Un compromiso mas grande y una asistencia mas concreta de las autoridades regionales y de los organismos del gobierno sobre todo con respecto a la reconversion profesional y la promoción de un medio ambiante empresarial propicio, deverián favorecer el desarrollo y la expansion de las PME en Polonia.

\section{Introduction}

La nécessité d'une adaptation rapide aux transformations qui s'effectuent actuellement dans l'économie mondiale peut constituer une chance pour les réformes polonaises. La complexité de ces réformes découlent surtout du fait qu'elles se passent dans une phase où le capitalisme contemporain connaît une transformation radicale. En effet, la deuxième révolution industrielle est terminée et, avec elle, une évolution contrôlée de la production, laquelle constituait un des traits caractéristiques de la croissance, des marchés relativement stables et une arri- 
vée régulière de la main-d'œuvre. À présent, ce sont de brusques changements et une forte turbulence de la production qui caractérisent l'économie mondiale. Comme une réponse à ces exigences de la mutation, on assiste à un accroissement du nombre et du rôle des petites firmes. Celles-ci sont souvent des « expérimentateurs » en introduisant de nouvelles productions sur le marché, en utilisant temporairement une main-d'œuvre supplémentaire et en facilitant la (re)formation professionnelle de celle-ci. Pour l'économie actuelle, les facteurs de production les plus importants deviennent :

- une main-d'œuvre hautement qualifiée ;

- des centres de recherche et des universités bien développées ;

- un environnement facilitant l'entreprenariat ;

- une qualité et un niveau de vie attrayant, etc.

L'objectif du présent article est de montrer les menaces et les obstacles fondamentaux à la restructuration de l'économie archaïque polonaise, face aux exigences imposées par la nouvelle économie mondiale. Lodz, seconde ville de Pologne, sert d'exemple ; l'état des investissements urbains et le type d'industries qui s'y trouvent exigent des transformations profondes.

\section{Les obstacles de base pour la restructuration de l'économie et pour le développement de nouvelles entreprises en Pologne}

\subsection{Les obstacles externes}

La crise des marchés traditionnels, plutôt stables tant du côté vente qu'achat, constitue, pour plusieurs entreprises polonaises, un obstacle pour leur développement industriel. Les marchés des anciens pays du COMECON étaient caractérisés par une structure traditionnelle de la demande avec une qualité de produits bien inférieure à celle des biens disponibles sur le marché mondial.

La nouvelle situation va contraindre les entreprises polonaises à des décisions draconiennes quant à leur restructuration et à leur adaptation aux nouveaux marchés. Le coût social sera grand et cette restructuration sera d'autant plus difficile que les entrepreneurs polonais manquent d'expérience et de connaissances nécessaires pour agir dans un marché libre. Par exemple, une expertise avancée en marketing et en gestion est indispensable ; elle nécessiterait sans doute une assistance institutionnelle et une aide organisationnelle du gouvernement. 
La récession actuelle de l'économie mondiale réduit les possibilités d'exportation des entreprises polonaises et limite les investissements étrangers. À cet égard, l'absence de technologies modernes et la qualité médiocre des produits qui en découle constituent un obstacle important. L'économie polonaise manifestera toujours une grande sensibilité aux modifications des prix internationaux énergétiques étant donné une compétitivité très faible de la production charbonnière polonaise. De plus, l'exportation de produits à valeur ajoutée très basse ne facilite pas les choses.

\subsection{Les obstacles Internes}

Du côté interne, un des obstacles est la faible réactivité des entreprises polonaises aux facteurs de l'environnement sociopolitique. L'industrie nationale manifeste une résistance relativement grande à l'innovation et à la réduction des coûts de production. Des facteurs de nature psychosociale et bureaucratique, dérivés du système d'économie centralisée, y contribuent pour une bonne partie. Un élément essentiel du développement est l'aptitude à courir des risques ; or, cette aptitude fait défaut dans les entreprises polonaises. Cette situation est tout à fait nouvelle en Pologne de l'après-guerre. L'esprit d'innovation, la capacité de prendre des risques, le recours à l'emprunt sont également bloqués par le système juridique existant.

L'ignorance générale des principes modernes de marketing et de gestion constitue un autre obstacle. La réaction type des entreprises à la modification de la demande est de diminuer la production ou de faire monter les prix. Elle se caractérise par des réactions à court terme qui découlent en partie de la mentalité des entrepreneurs et des ouvriers, mais elle relève également de l'absence de règles claires et cohérentes dans la gestion des entreprises (les critères de la privatisation, le système de crédits et d'impôts, le système douanier, etc.).

Un autre obstacle provient du manque d'institutions et d'organisations professionnelles qui assisteraient les entrepreneurs pour rencontrer les besoins du marché international, national ou local. Parmi les plus importantes d'entre elles, il faut mentionner les institutions financières, les organismes d'assurance, les cabinets de consultants, les firmes spécialisées en publicité, les firmes de capitaux de risque, les organismes régionaux et locaux de développement (création du matériel industriel, des réserves, etc.).

L'absence de relations ou des relations très peu développées entre la théorie et la pratique, ainsi qu'entre ces dernières et les institutions de recherche et développement compte aussi parmi les obstacles internes importants.

Une réglementation juridique claire dans le domaine de la propriété urbaine et dans celui de la reprivatisation des biens, dont le statut juridique n'a 
toujours pas été réglé, fait toujours défaut. On considère que cette situation, à propos de la propriété des biens dans plusieurs villes, constitue l'un des principaux obstacles au développement. Ceci freine les investissements, en particulier dans le centre des villes et réduit l'engagement des capitaux privés.

La pénurie de logements présente un autre obstacle majeur pour la restructuration de l'industrie polonaise. En effet, elle diminue considérablement la mobilité de la main-d'œuvre. Il en découle que la politique relative au logement décidera à long terme du développement économique et de l'acceptation par la société des réformes engagées. L'absence de règlements juridiques dans le domaine de l'aménagement spatial dans un sens large constitue un autre obstacle artificiellement créé, sous forme d'une offre réduite de terrains pour le bâtiment et de leur développement en infrastructure. Ceci entrave visiblement le développement urbain ; il est souvent impossible de définir les zones d'aménagement industriel dans les villes.

Un autre obstacle découle du mauvais fonctionnement des pouvoirs publics territoriaux. Ces derniers se trouvent dans une phase d'apprentissage et d'expérimentation dans la mise en place de règlements juridiques objectifs et clairs, lesquels sont présentement souvent obscurs. Leur bon fonctionnement exercera une forte influence sur l'aménagement futur, indépendamment du fait que la commune présente ou non un potentiel de développement.

Un penchant excessif des autorités locales et de la population pour l'égalitarisme peut devenir une menace pour les réformes et les transformations rapides de l'économie nationale. Il existe là une forte résistance sociale contre toute reproduction des classes sociales et contre des différences dans les salaires et dans les conditions de vie. Cette question acquiert une complexité particulière dans les conditions de vie d'une société postcommuniste.

L'absence d'infrastructure représente une autre barrière dont l'importance est difficile à évaluer. En Pologne, on trouve un système de communication et de télécommunication archaïque, un faible réseau de transports interrégionaux (routes, chemin de fer, avion); il en va de même pour les autoroutes internationales, dont le réseau est peu développé.

De plus, les constructions illégales représentent un phénomène qui devient un véritable problème à l'heure actuelle. Celles-ci prolifèrent dans les zones suburbaines et dans les zones d'attraction de grandes agglomérations faisant ainsi obstacle au développement des villes. Ces constructions sont caractéristiques des zones où l'on observe une concurrence entre les fonctions urbaines (loisirs, logements, production, etc.) et les fonctions agricoles. Un fort démembrement des terres cultivées et un nombre important de travailleurs à double profession (agriculteurs-ouvriers) sont deux caractéristiques de ces espaces. On y trouve des gens qui construisent massivement et d'une manière 
illégale des résidences secondaires, des bâtiments pour une PME, des garages ; certains reconstruisent illégalement leur maison, etc. Sur ces terrains, on installe un nombre relativement grand d'ateliers pour l'artisanat, des entreprises de production et des services. Ces constructions sont faites hors des limites administratives urbaines pour éviter de payer les impôts urbains. On crée ainsi un espace où fonctionne un circuit économique secondaire (informel). Pour mettre fin à ce phénomène, il faut changer le droit en vigueur et, en particulier, les règlements qui concernent et décident de la répartition spatiale.

\section{Opportunités et obstacles pour le développement des petites et moyennes entreprises}

L'expansion des petites entreprises constitue l'une des voies possibles menant à la restructuration de l'économie et une méthode simple pour agrandir la part de contrôle privé. Cela est d'autant plus important que les petites firmes jouent toujours un rôle marginal dans l'économie polonaise. Les avantages des petites entreprises sont leur flexibilité quant à la demande, leur aspect inventif élevé, la création d'emplois nouveaux et, finalement, une chance pour les entrepreneurs de se faire valoir.

Les transformations observées ces dernières années dans la structure industrielle des pays développés n'ont d'ailleurs pas entraîné l'élimination de petites firmes. Dans l'économie polonaise, la part et l'importance économique de petites firmes étaient secondaires. Par exemple, dans le secteur manufacturier en 1985, la proportion des entreprises de moins de 200 salariés ne représentait qu'environ $8 \%$, et celle des entreprises de moins de 50 employés dépassait à peine $3 \%$ du nombre général des emplois industriels. D'où l'importance mineure des entreprises de petite taille dans la production et dans l'emploi en Pologne. Cette faiblesse dans le secteur des PME suppose une nouvelle politique économique pour favoriser l'entreprenariat.

Les principes de base pour une mise en place des activités par les entrepreneurs potentiels sont :

1. avoir une idée relative à un produit et/ou un service ;

2. disposer d'un capital ;

3. posséder un savoir-faire professionnel, technique et économique ;

4. avoir un local adapté ou adaptable ;

5. avoir le sens de la gestion ; 
6. avoir des prédispositions psychologiques nécessaires ;

7. savoir se procurer les moyens de production.

Par rapport aux principes indispensables pour la création de sa propre entreprise, on peut identifier diverses entraves. On peut les diviser selon les groupes suivants :

- les obstacles qui résultent du manque de capitaux et de la difficulté de disposer d'un local ;

- les obstacles liés à l'esprit d'entreprise, tel le manque d'idée sur les biens ou le(s) service(s) à offrir, l'inaptitude à accepter le risque, le désir de conserver la sécurité sociale... Tous ces facteurs contribuent à ce que l'on appelle « l'esprit d'entreprise » qui peut considérablement freiner ou au contraire stimuler le développement des firmes ;

- les obstacles reliés à la formation, tel le manque de connaissances professionnelles et administratives, le manque d'expérience, l'impossibilité d'évaluer l'évolution d'une entreprise ;

- les obstacles systémiques liés à la méfiance par rapport à la stabilité du régime sociopolitique et à des solutions juridiques favorisant le développement des PME.

Au tableau 1, on a évalué le type et la force des principaux obstacles au développement de l'entreprenariat soit potentiel, soit actuel, selon le type d'entrepreneurs. 
TABleau 1

Les obstacles les plus importants pour la mise en place et/ou le développement d'une petite entreprise en Pologne

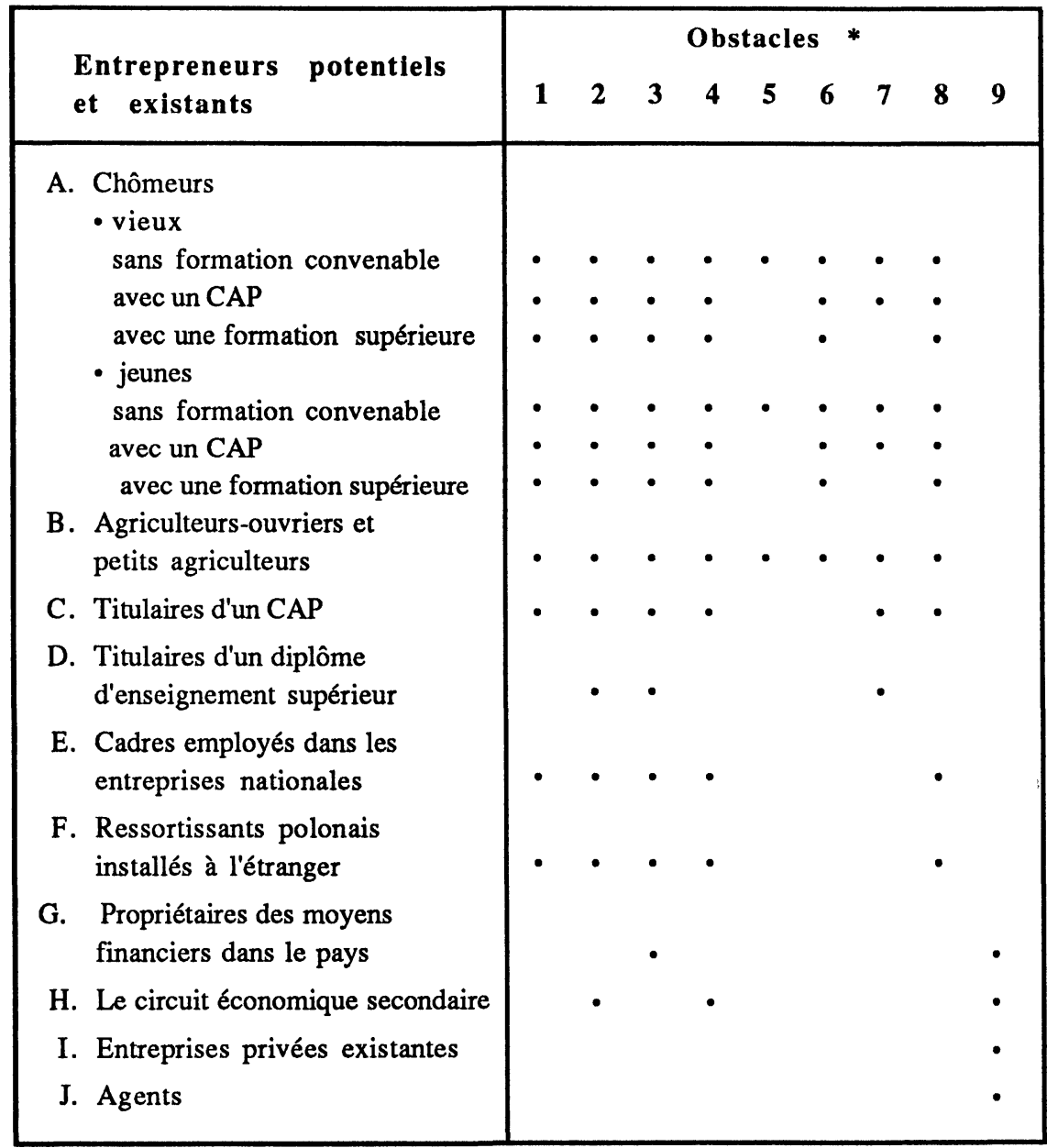

* 1. manque de capital ;

2. manque d'idée sur un produit et/ou un (des) service(s);

3. pénurie de locaux ;

4. ignorance en management ;

5. manque de connaissance professionnelle ;

6. recherche de la sécurité sopciale (argent épargné et biens propres destinés à la sérucité sociale);

7. incapacité à évaluer les profits à tirer d'un investissement, à la mise en place d'une activité ;

8. inaptitude à courir un risque ;

9. méfiance à l'égard de la stabilité du système.

Source : J. JETTMAR, Les obstacles les plus importants pour la mise en place etlou le développement d'une petite entreprise, Centrum Promocji, Gdansk, 1990. 
L'esprit d'entreprise chez les entrepreneurs potentiels doit être avant tout encouragé et développé chez les quatre derniers groupes, soit les ressortissants polonais, les propriétaires des capitaux établis dans le pays, les représentants du circuit économique secondaire, ou encore dans les entreprises privées existantes. Néanmoins, les entrepreneurs de ces groupes manifestent en même temps une méfiance face aux perspectives de stabilité du régime. Cet obstacle ne peut être levé qu'avec l'établissement de réponses claires, évidentes et inébranlables. D'autant plus que les possibilités de ces groupes d'entrepreneurs sont remarquables, comme en témoigne, par exemple, une évaluation du capital dans le circuit secondaire polonais dont le niveau, estimé en 1986, varie entre 941 et 1,029 milliard de zlotys.

Un autre trait caractéristique de cette analyse est que le nombre d'obstacles identifiés est plus faible dans les groupes ayant une expérience dans une activité donnée. C'est un fait prouvé que, d'habitude, les gens qui ont acquis une expérience dans une branche donnée délaissent ou refusent souvent le statut de salariés pour ouvrir leur propre entreprise, laquelle fait souvent concurrence à celle de leur ancien employeur.

\section{Opportunités et obstacles pour les transformations structurelles à Lodz}

Lodz est une ville industrielle où dominent les industries dites légères, surtout textiles. Elle est née grâce au développement industriel dynamique de la fin du xIxe siècle et du début du xxe siècle. La fonction industrielle est même l'une des fonctions principales de cette ville. Toute la politique de son développement économique après la Deuxième Guerre mondiale a été marquée par l'agrandissement et la modernisation des établissements qui s'y trouvaient.

Lodz n'a pas subi de dégâts au cours de cette guerre. Elle en est donc sortie avec un aménagement urbain presque intact, mais aussi avec tout un héritage de conséquences négatives du développement très rapide du xIxe siècle.

Dans la période de l'après-guerre, les problèmes techniques et sociaux de la ville les plus urgents ont pu trouver, dans une certaine mesure, une solution, mais ce ne fut pas le cas dans tous les domaines. La situation économique du pays a privé la ville de diverses possibilités ou de conditions nécessaires à un développement harmonieux. La lutte contre la pénurie de logements et la satisfaction des besoins courants ont provoqué une expansion de la ville sur les terrains disponibles pour un meilleur aménagement et a augmenté les disparités toujours croissantes entre l'infrastructure du centre-ville et les nouveaux quar- 
tiers. La principale conséquence de ce processus a été une diminution des standards des services urbains à Srodmiescie (le centre-ville). Dans la périphérie de la vieille ville, l'aménagement s'est graduellement transformé, mais, malheureusement, de façon presque toujours partielle. Les zones centrales de la ville se sont avérées les plus « résistantes » aux transformations, vu leur forte densité.

Un mauvais état de la ville après la Deuxième Guerre mondiale, un manque d'investissements pour soutenir un fonctionnement normal et la stratégie de développement qui a été acceptée ont créé de nombreuses disparités tout en entraînant un processus négatif sur l'aménagement spatial de la ville. Les vieux problèmes n'ont pas trouvé leurs solutions et de nouveaux problèmes ont surgi.

Les phénomènes observés et leurs conséquences peuvent être caractérisés de la manière suivante :

- le très faible aménagement de la zone extérieure de la ville ;

- un déplacement important de la population dans les nouveaux immeubles ;

- une diminution permanente de la population du centre-ville ;

- des modifications dans la structure de la population de la zone centrale : les vieux y restent (pour des facteurs économiques ou à cause des coûts du déménagement) et les jeunes y viennent en considérant leurs logements comme temporaires ;

- d'où une faible activité de la population du centre : les vieux ne s'engagent pas dans des activités sociales et les jeunes ne s'y intéressent pas. L'analyse des postulats (problèmes) aux élections communales est intéressante à cet égard : environ $80 \%$ de ces postulats concernent l'exploitation quotidienne des logements gérés par les concierges, et, éventuellement, par les services d'urgence. Il n'y a que très peu de postulats relatifs aux problèmes plus substantiels pour ce quartier ;

- le phénomène de la ségrégation sociale progresse : les pauvres restent au centre (où les loyers sont les plus bas et où l'on trouve la plus grande pathologie sociale);

- la dégradation des logements s'accélère ; les modernisations et les remises en état font défaut ;

- les relations de propriété (les logements d'État et privés) liées au loyer bon marché et à la structure sociale de la population bloquent les investissements nouveaux ;

- les standards des logements diffèrent : dans la zone extérieure de la ville, on trouve des logements équipés en infrastructure, mais avec des 
surfaces réduites, et dans la zone centrale, il y a les logements avec des surfaces considérables, mais sans équipement et mal conservés ;

- les réserves des terrains aménageables sont très petites au centre et plus grandes, quoique sans infrastructure, dans la zone extérieure ;

- le tertiaire où les services sont concentrés à $70 \%$ dans la zone centrale, alors que les constructions nouvelles ne sont pas assez desservies tant du côté de la capacité tertiaire que de son exploitation ;

- la croissance des transports individuels et collectifs de communication urbaine fait défaut et cause des parcours de transit par le centre ;

- les transports publics sont insuffisants et inégalement répartis ;

- les exigences concernant les standards des logements sont de plus en plus grandes ;

- les constructions nouvelles diminuent et la structure des logements n'est pas adaptée à la demande ;

- la répartition de l'habitat est spatialement et structurellement inégale ;

- l'état de l'environnement est très grave et même critique ;

- les activités de modernisation ne sont pas suffisantes;

- la reconstruction du centre n'est pas correcte et conséquente ; on observe un éparpillement des ressources et les programmes généraux de cette reconstruction font défaut.

\subsection{La localisation des Industries dans la ville}

L'ancien aménagement spatial industriel a finalement subsisté, avec quelques petites modifications, jusqu'à la moitié des années 60 . Sa principale caractéristique était une grande concentration des établissements (surtout dans l'industrie du textile) dans la partie centrale de la ville.

Cette concentration excessive d'établissements industriels sur une surface limitée s'est manifestée d'une manière particulièrement frappante avec les modifications des limites administratives de la ville et de sa répartition en quartiers dans l'après-guerre. L'agrandissement de la ville fait en sorte que presque toutes les industries se sont retrouvées dans deux quartiers seulement : Srodmiescie (au centre-ville) et Polesie. Les terrains du premier quartier couvrait presque entièrement l'espace de l'expansion du Lodz industriel au xIxe siècle et c'est cette zone qui a une influence décisive sur ce que l'on appelle «l'image de la ville ». La division industrielle de la ville en quartiers peut être illustrée au schéma 1. 


\section{SCHÉMA 1}

La caractéristique des industries de Lodz. La répartition en quartiers (part totale en pourcentage)

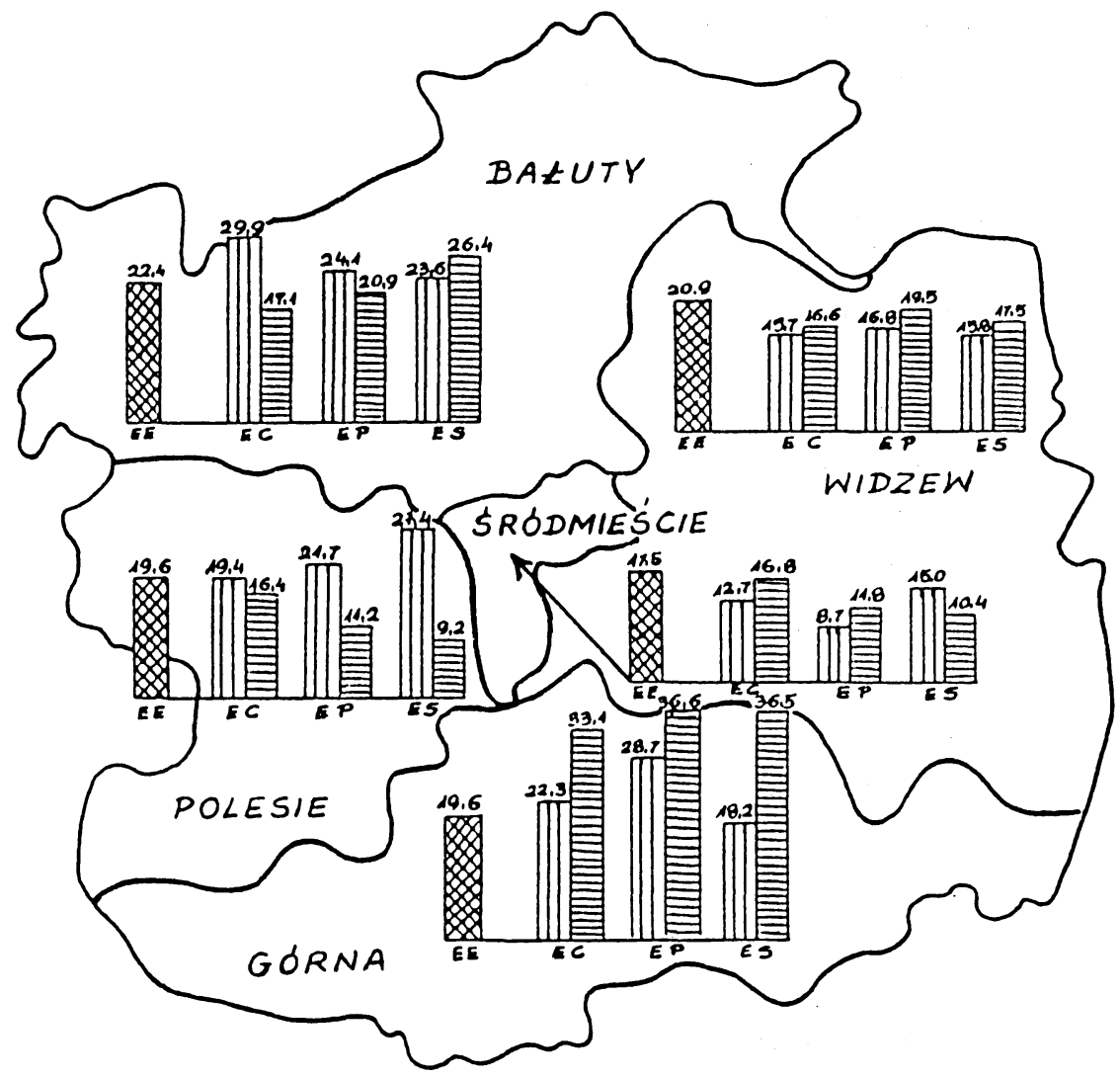

entreprises d'État - personnes employées en \% en 1988

nombre d'entreprises mises en place - petites firmes privées en 1990

nombre d'entreprises liquidées et suspendues - petites firmes privées en 1990

EE entreprises d'État (totales);

EC entreprises commerciales ;

EP entreprises de production ;

ES entreprises de services. 
Dans la seconde moitié des années 60 , les transformations remarquables se font voir dans la répartition dans l'espace urbain. L'agrandissement et la modernisation de la ville en sont la cause. Ce programme a pour objectif la liquidation des vieux bâtiments particulièrement nuisibles et la construction de nouvelles entreprises, surtout à la périphérie de la ville. Ces entreprises devaient être localisées exclusivement dans les terrains spécialement aménagés dans des quartiers industriels.

En conséquence, le rôle du centre-ville en tant que quartier plus industrialisé diminue considérablement. Il en va de même pour la structure des industries dans les quartiers traditionnels. L'industrie légère domine toujours, mais sa domination diminue lentement et systématiquement. Plusieurs nouvelles entreprises voient le jour ; mais leur localisation se fait en dehors du centre-ville où ne restent que les anciens établissements. En outre, les modernisations sont de faible envergure. Dans le passé, les investissements se concentraient dans les industries situées dans les quartiers de Widzew et de Gorna. On peut voir les caractéristiques des entreprises de production et leur répartition au tableau 2.

\section{TABLEAU 2}

\section{La caractéristique de l'industrie de Lodz selon la répartition par quartiers en 1988}

\begin{tabular}{|c|c|c|c|c|c|c|c|c|}
\hline \multirow[t]{2}{*}{ Quartiers } & \multicolumn{2}{|c|}{ Surface } & \multicolumn{2}{|c|}{ Population } & \multicolumn{2}{|c|}{ Pourcentage } & \multicolumn{2}{|c|}{ Milliers de zlotys } \\
\hline & $\mathrm{Km}^{2}$ & $\%$ & Nombre & $\%$ & $\begin{array}{l}\text { Emploi } \\
\text { (a) }\end{array}$ & $\begin{array}{c}\text { Valeur } \\
\text { brute } \\
\text { des } \\
\text { biens } \\
\text { durables }\end{array}$ & $\begin{array}{l}\text { Équipe- } \\
\text { ment } \\
\text { technique } \\
\text { du travail } \\
\text { (b) }\end{array}$ & $\begin{array}{l}\text { Rende- } \\
\text { ment du } \\
\text { travail }\end{array}$ \\
\hline Bahuty & 63,7 & 29,8 & 245277 & 29,1 & 22,4 & 19,3 & 1346,6 & 544,5 \\
\hline Goma & 52,6 & 24,5 & 199423 & 23,6 & 19,6 & 25,3 & 2026,9 & 618,9 \\
\hline Polesie & 37,8 & 17,6 & 172175 & 20,4 & 19,6 & 16,2 & 1293,9 & 633,0 \\
\hline Srodmiescie & 6,8 & 3,2 & 100931 & 11,9 & 17,5 & 12,5 & 1119,4 & 389,3 \\
\hline Widzew & 53,4 & 24,9 & 127140 & 15,0 & 20,9 & 26,7 & 2002,2 & 501,9 \\
\hline Lodz & 214,3 & 100,0 & 844946 & 100,0 & 100,0 & 100,0 & 1622,9 & 540,5 \\
\hline
\end{tabular}

(a) emploi moyen dans l'année - (b) valeur brute des biens durables sur un salarié

(c) quotient de la valeur de la production vendue exprimée en prix courants de vente et de l'emploi moyen dans l'année

Source : J. Lus. Travaux de recherche du Département d'Économie du Développement des villes, Lodz 1987-1989 et études personnelles. 
Dans la structure industrielle de Lodz dominaient et dominent toujours les grandes entreprises. Mais depuis 1989, on peut constater une diminution du nombre de grandes entreprises. Ces changements ont comme source la crise que vit l'industrie légère. Depuis 1988, la production dans ce secteur va en diminuant et une réduction générale des emplois peut être observée.

Ces changements n'ont cependant pas une grande importance pour le niveau de concentration des industries de Lodz. Comme l'indiquent les données présentées au tableau $3,55,8 \%$ des emplois, $49,5 \%$ de la valeur de la production industrielle vendue et même $64,2 \%$ des biens durables appartiennent aux grandes entreprises. L'importance et le rang de petites entreprises (moins de 200 employés) demeurent faibles. Elles procurent $6,6 \%$ de l'emploi dans le secteur industriel public, ne disposant que de 5,0\% des biens durables et ne réalisent que $4,2 \%$ de la valeur de la production.

TABLEAU 3

Niveau de concentration de l'industrie de Lodz en 1990

\begin{tabular}{|l|c|c|c|c|}
\hline $\begin{array}{c}\text { Dimension } \\
\text { de l'entreprise } \\
\text { mesurée } \\
\text { par le nombre } \\
\text { de personnes } \\
\text { embauchées }\end{array}$ & $\begin{array}{c}\text { Nombre } \\
\text { d'entreprises }\end{array}$ & $\begin{array}{c}\text { Personnes } \\
\text { embauchées }\end{array}$ & $\begin{array}{c}\text { Production } \\
\text { vendue }\end{array}$ & $\begin{array}{c}\text { Valeur brute } \\
\text { des biens } \\
\text { durables }\end{array}$ \\
\hline & & & & \\
Jusqu'à 100 & 33 & 1,4 & 1,4 & 0,4 \\
$101-200$ & 45 & 5,2 & 2,8 & 3,6 \\
$201-500$ & 78 & 14,9 & 23,3 & 11,0 \\
$501-1000$ & 51 & 22,7 & 23,0 & 20,8 \\
$1001-2000$ & 33 & 28,1 & 26,0 & 32,7 \\
Plus de 2001 & 16 & 27,7 & 23,5 & 31,5 \\
& & & & \\
\hline
\end{tabular}

Source : J. Lis. Travaux de recherche, Lodz 1991 et études personnelles.

Ces petites unités disposent d'équipements et de bâtiments moins modernes. Cependant, elles ont le meilleur indice quant à la productivité des biens durables (tableau 4), sans que l'on puisse toutefois noter une différence importante dans le rendement du travail. Il semble donc que leurs activités soient gérées de manière plus rationnelle, alors que les indices pour les plus grandes entreprises ne permettent pas une évaluation très positive de leurs activités. 
TABLEAU 4

Taille des entreprises et relations économiques de base de l'industrie de Lodz en 1990

\begin{tabular}{|l|c|c|c|}
\hline $\begin{array}{c}\text { Dimension } \\
\text { des entreprises } \\
\text { mesuré }\end{array}$ & $\begin{array}{c}\text { Rendement } \\
\text { du travail } \\
\text { en million } \\
\text { par le nombre } \\
\text { de personnes } \\
\text { embauchées }\end{array}$ & $\begin{array}{c}\text { Equipement } \\
\text { technique } \\
\text { du travail } \\
\text { en million } \\
\text { de zlotys } \\
\text { (a) }\end{array}$ & $\begin{array}{c}\text { Productivité } \\
\text { des biens } \\
\text { durables } \\
\text { en zlotys } \\
\text { (c) }\end{array}$ \\
\hline $\begin{array}{l}\text { Jusqu'à } 100 \text { pers. } \\
101-200\end{array}$ & 96,7 & 61,0 & 1386 \\
$201-500$ & 49,3 & 144,3 & 341 \\
$501-1000$ & 128,7 & 151,6 & 849 \\
$1001-2000$ & 89,8 & 187,3 & 479 \\
Plus de 2001 & 94,4 & 238,6 & 396 \\
& 97,0 & 232,5 & 417 \\
\hline
\end{tabular}

(a) valeur de la production au prix courant par rapport à l'emploi moyen

(b) valeur brute des biens durables par rapport à l'emploi moyen

(c) valeur de la production vendue au prix courant sur $1000 \mathrm{ZL}$ de la valeur brute des biens durables

Source : J. Lis. Travaux de recherches 1991, études personnelles.

Les industries de Lodz connaissent présentement de grandes difficultés. On évalue qu'avant 1992, $65 \%$ des entreprises se trouveront en faillite. L'indice du chômage dans cette région atteint déjà $20 \%$. On admet en général qu'il faille trouver d'autres formes d'activités. Celles-ci doivent être trouvées dans la privatisation générale des entreprises d'État existantes, dans la diminution de leur taille, dans la promotion et le développement des petites firmes privées.

\section{L'expansion de petites firmes privées. Tendances et localisation}

En 1990, on a réalisé à Lodz plusieurs recherches concernant le développement des entreprises privées. Ces recherches portaient sur les établissements divisés en entreprises artisanales, manufacturières, commerciales et de services. On a également procédé à une analyse de leurs localisations dans la ville et de leur « mouvement », c'est-à-dire du processus de suspension, de liquidation ou de création (tableau 5). 
TABleau 5

Nombres des entreprises privées liquidées et mises en place à Lodz en 1990

\begin{tabular}{|c|c|c|c|c|c|c|}
\hline \multirow[t]{2}{*}{ Quartiers } & \multicolumn{2}{|c|}{$\begin{array}{l}\text { Entreprises } \\
\text { commerciales }\end{array}$} & \multicolumn{2}{|c|}{$\begin{array}{l}\text { Entreprises } \\
\text { de production } \\
\text { et artisanales }\end{array}$} & \multicolumn{2}{|c|}{$\begin{array}{l}\text { Entreprises } \\
\text { de services }\end{array}$} \\
\hline & $\begin{array}{c}\text { Liquidées } \\
\text { et } \\
\text { suspendues }\end{array}$ & $\begin{array}{l}\text { Mises } \\
\text { en } \\
\text { place }\end{array}$ & $\begin{array}{c}\text { Liquidées } \\
\text { et } \\
\text { suspendues }\end{array}$ & $\begin{array}{l}\text { Mises } \\
\text { en } \\
\text { place }\end{array}$ & $\begin{array}{c}\text { Liquidées } \\
\text { et } \\
\text { suspendues }\end{array}$ & $\begin{array}{l}\text { Mises } \\
\text { en } \\
\text { place }\end{array}$ \\
\hline Baluty & 1193 & 3673 & 4077 & 1935 & 2848 & 534 \\
\hline Goma & 2302 & 2733 & 7145 & 2309 & 3942 & 412 \\
\hline Polesie & 1145 & 2384 & 2186 & 1741 & 996 & 620 \\
\hline Srodmiescie & 1167 & 1555 & 2294 & 700 & 1126 & 339 \\
\hline Widzew & 1157 & 1929 & 3800 & 1350 & 1897 & 360 \\
\hline Lodz & 6964 & 12274 & 19502 & 8305 & 10809 & 2267 \\
\hline
\end{tabular}

Source : études personnelles

Parmi les établissements nouvellement créés, on trouve surtout des petits commerces. Cette activité domine dans tous les quartiers de Lodz. Les activités de services, comparées aux activités commerciales et artisanales, constituent une partie négligeable. Parmi les entreprises liquidées ou suspendant leurs activités, les établissements artisanaux et les entreprises industrielles constituaient plus de $52,0 \%$. Vingt-neuf pour cent des établissements dans ce groupe étaient des entreprises de services.

La période de juillet-août 1990 a été une période cruciale en ce qui concerne l'intensité du « mouvement » des entreprises. On y trouve le plus grand nombre d'entreprises qui ont mis fin à leurs activités ; par contre, après cette période, on remarque des enregistrements massifs de nouvelles entreprises développant souvent de nouvelles activités ou présentant un nouveau profil d'entreprise.

On peut supposer qu'un processus systématique visant l'adaptation des entreprises aux nouvelles conditions économiques aurait commencé, par exemple, par un accroissement d'entreprises créées à la fin de 1990 et par une réduction du rythme de diminution du nombre d'entreprises liquidées et/ou suspendues. 
Les enquêtes réalisées auprès des personnes qui fermaient leurs entreprises a permis de dégager certaines caractéristiques de ce groupe d'entrepreneurs. Dans la plupart des cas, ce sont des vieux propriétaires d'entreprises qui ont œuvré pendant des années dans une même branche industrielle. Ils vendaient leurs produits soit aux entreprises d'État, soit aux clients individuels (produits d'usage commun). La crise dans le secteur industriel public, les importations de produits occidentaux de bien meilleure qualité, tout comme la baisse de la demande due à l'appauvrissement de la société ont largement contribué à la perte de clients, c'est-à-dire aux possibilités de ventes. Ces entrepreneurs, incapables d'adaptation rapide aux conditions nouvelles, $n$ 'ont pas pu faire le changement de profil de leurs entreprises. Ces propriétaires n'avaient pas les grands capitaux qui leur auraient permis de moderniser rapidement leurs entreprises. Le taux très élevé du crédit leur rendait cette forme d'assistance impossible. Paradoxalement, les entreprises qui n'ont pas contracté de dettes pendant la période de 1989-1990 se trouvaient dans une meilleure position financière. Le taux élevé d'inflation et du crédit qui en découlait a surpris beaucoup d'entrepreneurs et les a poussés à la faillite.

L'absence d'une idée directrice générale de développement et, par conséquent, l'absence de stabilité a été une autre cause du nombre relativement grand de liquidations et de suspensions d'activités. Les entrepreneurs cherchaient alors des profits rapides ; $s$ 'ils ne les obtenaient pas en un court laps de temps, ils changeaient de production ou, le plus souvent, passaient au secteur commercial. Cette dernière tendance a été négative pour l'économie parce qu'elle a retardé la restructuration industrielle et a freiné la circulation de capital dans la production. En effet, les firmes commerciales vendent surtout les produits importés.

Certains facteurs ont contribué à la domination des entreprises commerciales, entre autres :

- un marché national ouvert et dépourvu de nombreux produits de base ;

- des prix plus compétitifs dans le cas d'une coopération avec des firmes étrangères ;

- les facilités offertes par les règlements douaniers pour les importations et la vente de certaines marchandises ;

- la prolifération de nouveaux magasins due à la privatisation. Celle-ci a d'abord porté sur les activités commerciales, là où les autorités locales pouvaient exercer la plus grande influence ;

- la naissance de grands stocks privés, phénomène nouveau à l'échelle nationale, ce qui a entraîné des activités commerciales à une plus grande échelle. 
Les activités tertiaires ou de services et leur faible importance par rapport aux besoins existants découlent aussi des conditions économiques nouvelles. De nombreux services offerts sont trop chers pour le client moyen. De plus, il y en a beaucoup auxquels les gens ne sont simplement pas habitués, ce qui a poussé un certain nombre de firmes nouvelles à liquider leurs activités, faute de demande. Ceci indique que plusieurs activités ont été mises en place sans analyse économique suffisante. Ces firmes ont été créées souvent en suivant les principes du « il faut faire quelque chose » et « l'on réussit par hasard ». Ces entrepreneurs disposaient de moyens trop modestes et n'avaient aucune expérience en management. En plus, ils se sont engagés dans des activités risquées sans capitaux suffisants. De plus, le soutien des organes du gouvernement et des autorités locales étaient à peine visible.

Les entreprises qui fonctionnent et qui ont une chance de survivre sont en général dirigées par des personnes âgées d'environ 40 ans et qui, à $90 \%$, ont une formation supérieure. Dans ce groupe, on peut distinguer deux catégories d'entrepreneurs. La première catégorie se compose de personnes qui travaillaient auparavant dans les entreprises d'État et qui ont mis en place une activité conforme à la profession qu'elles exerçaient dans ces entreprises. Ces entrepreneurs profitent de leur expérience vécue jusqu'alors, de leur formation professionnelle et, surtout, de leurs contacts dans le pays quant à la coopération et la vente. L'autre groupe comprend des entrepreneurs qui ont une idée intéressante, qui ont eu l'occasion de passer quelque temps à l'étranger ou qui ont des contacts avec les ressortissants polonais établis à l'étranger ; ces derniers les aident à financer et à vendre leurs produits.

Ces nouvelles entreprises sont en général relativement bien équipées en matériel moderne et, surtout, en appareils de télécommunication (téléphones, télécopieurs, ordinateurs, etc.). Leurs activités sont peu liées aux activités traditionnelles industrielles de la ville et de la région ; elles profitent peu du potentiel local, surtout à cause d'une régression croissante de l'industrie traditionnelle, d'une diminution de la production et du déclin des relations avec les autres entreprises.

Les nouveaux entrepreneurs cherchent avant tout les contacts avec les entreprises étrangères. La localisation de ce type d'entreprises est aussi caractéristique ; les plus commerciales se retrouvent surtout au centre de la ville. L'une des causes de cette situation est la privatisation qui a porté dans un premier temps sur le commerce et les services. Les entreprises de ce secteur étaient situées surtout dans le centre-ville; d'où leur maintien en grand nombre dans cette zone. Les entreprises de production manufacturière et plusieurs PME de service sont localisées dans les autres quartiers de Lodz. Ces activités ont lieu très souvent près des domiciles ou même dans les logements de leurs proprié- 
taires. Dans le plupart des cas, il s'agit de propriétaires de maisons familiales localisées dans la périphérie de la ville. Il semble que cette tendance se poursuivra dans l'avenir. Le commerce et les services continueront à être orientés vers le centre de par leur nature ; d'autant plus que cela s'harmonise avec le programme de la remise en état de la partie centrale de la ville. Les activités industrielles pourront profiter des bâtiments laissés par les entreprises d'État liquidées. Après les travaux de rénovation, ces bâtiments pourraient servir de parcs industriels ou de réserves. Évidemment, ce processus exige une participation active des autorités régionales et locales.

\section{Conclusion}

L'analyse des opportunités et des obstacles du développement économique de la restructuration des industries à Lodz que nous avons faite indique l'importance et le poids de cette question pour l'économie nationale polonaise et, en particulier, pour les villes qui ont conservé une structure archaïque d'industries, dont Lodz est un exemple. Le processus est bien démarré ; mais il semble exiger un engagement plus grand et une assistance plus concrète des autorités régionales et des organes du gouvernement. La restructuration de cette région entraîne à coup sûr des conséquences sociales majeures : une baisse dans les standards des logements, le chômage et la perte de sécurité sociale. Dans le cas de la disparition des entreprises d'État, la seule solution est la substitution par les entreprises privées. Pour l'instant, le rôle de ces dernières dans la création d'emplois demeure très faible, d'autant plus que les nouvelles activités ne sont pas liées à l'industrie traditionnelle; les employeurs cherchent donc d'autres spécialistes que ceux disponibles sur le marché du travail. Ceci entraîne un paradoxe : malgré le chômage croissant, les entreprises ont du mal à trouver des travailleurs ayant des qualifications recherchées.

La reconversion professionnelle constitue sûrement l'une des tâches les plus urgentes des autorités locales. Outre les conditions externes comme le développement de la demande ou le système juridique, l'état de la ville de Lodz, avec le manque des moyen pour soutenir les investissements indispensables ou une structure spatiale vicieuse, constitue un obstacle pour le développement de l'esprit d'entreprise dans cette ville. Lodz, malgré son histoire, malgré ses centres universitaires et instituts de recherche, manque de ce que l'on appelle « l'environnement entrepreneurial ». Ceci impose aux autorités de la ville le devoir d'engager une campagne de publicité en faveur des PME, de promouvoir de nouvelles activités et de créer un cadre institutionnel et juridique favorisant la création de nouvelles entreprises. 


\section{Bibliographie}

Cher yl, A. Farr (1984), «Shaping the Local Economy », Current Perspectives on Economic Development, International City Management Association, Washington D.C..

JETTMAR, J. (1990), « Les plus importants obstacles pour une mise en place et/ou développement d'une petite entreprise ", Centrum Promocji, Gdansk.

Jetrmow, J. (1990), « Le développement de petites firmes », Revue d'Organisation $\mathrm{N}^{\circ} 10$.

Jewtuchowicz, A. (1991), «Les conditions de restructuration de l'industrie dans la région de Lodz », Travaux de recherche de l'Université de Lodz, 1991.

JEWTUCHOWICZ, A. (1991), «Les opportunités et les obstacles pour les transformations structurelles à Lodz », Travaux de recherche de l'Université de Lodz.

LIs, J. (1991), « Les conditions de la création de PME dans la région de Lodz », Travaux de recherche de l'Université de Lodz.

Mengin, J. (1989), Guide du développement local et du développement social, Éd. L'Harmattan.

Travaux de recherche du Département d'économie du développement des villes, 1989-1991, Lodz. 